\title{
Concepts of risk in dental public health
}

Brian A. Burt

Department of Epidemiology, School of Public Health, University of Michigan, Ann Arbor, MI, USA

Burt BA. Concepts of risk in dental public health. Community Dent Oral Epidemiol 2005; 33: 240-7. (C) Blackwell Munksgaard, 2005

Abstract - The purpose of this paper is to review the concepts of risk as we use them today in dental public health practice, and to suggest that we should broaden our view of risk. Use of terms like risk factor in the literature can be quite vague, and it is recommended that a clear definition of that and related terms be adhered to. A broader view of risk in dental research would take in the concepts of social determinants of health and population health. While some progress has been made in our understanding of these issues, better knowledge would give the public health administrator more readily available information to use in program planning. The skewed distribution of caries in the highincome countries has led to the emergence of targeted prevention programs toward those considered to be at high risk. In public health programs, targeting at the individual level is not practical: the risk assessment methods are not yet sufficiently precise, and even when individuals are identified there are practical problems with schools and with the children themselves. (For private practice, however, high-risk child patients can be identified as those with at least one approximal lesion in permanent teeth.) For public health purposes, an argument is made for geographic targeting, i.e. identification of areas of social deprivation where whole schools or school districts can be targeted. Geographic targeting is something between individual targeting and whole-population approaches. Ideally, geographic targeting would supplement population measures like water fluoridation and dental health education. Examples of geographic targeting from Ohio and New York are presented as illustrations.

Key words: geographic targeting; high risk; population health; risk; risk factor; risk indicator; social determinants; targeting

Brian A. Burt, Department of Epidemiology, School of Public Health, University of Michigan, 109 Observatory Street,

Ann Arbor, MI 48109-2029, USA

Tel: 734-764-5478

Fax: 734-764-3192

e-mail: bburt@umich.edu

Submitted 14 September 2003; accepted 14 February 2005
Risk is the probability that an event will occur. In epidemiology, this is expanded to include a time frame, and hence risk becomes the probability that a particular event will occur within a given period of time (1). The term 'risk' is most often used to express the probability that a particular outcome will occur following a particular exposure, and it usually implies a bad outcome, i.e. disease or mortality, rather than a good one. (We do not much use an expression like '... risk of good health.') We are not interested in estimations when the risk is zero (i.e. when a particular outcome can never happen), nor when it is $100 \%$ (i.e. when a particular outcome must happen), but we are interested when the risk lies somewhere between those two extremes.

In public health practice, the concept of risk has been around since epidemiology first emerged as a science in the 19th century. Indeed, the core of the research effort in epidemiology down the years has been identifying and quantifying risk in one form or another. Absolute risk can be useful, although we most commonly deal with relative risk (or odds ratio), which estimates the probability of a particular outcome among exposed persons relative to the outcome among the nonexposed.

In dental public health practice, the concept of measuring and assessing risk, which means confronting the varying susceptibilities to the oral diseases, has only arrived more recently. One does not have to go back very far in time to reach a period when caries was almost universal among children in the high-income countries. There was little concept of risk at this time, for the probability of caries was seen to be close enough to $100 \%$. It is the decline in caries experience at population levels that has made it evident that 
today some children do not get the disease at all, some get it only to a minor degree, while others suffer badly from it. The natural question of why do some get the disease and others do not has brought the concept of risk into caries prediction. When data are available for a whole population, risk can be quantified precisely, but this is an unusual situation only possible with the best surveillance systems. When samples are involved, as they nearly always are, some estimation is needed.

This symposium deals with various aspects of caries risk. The purpose of this paper is to review the concepts of risk as we use them today in dental public health practice, and to suggest that we should broaden our view of risk to include social determinants of health and population health. Discussion of risk factors for caries leads us into targeting for prevention, and the idea of geographic targeting, as something between a wholepopulation strategy and targeting of individuals, will be presented.

\section{Current definition of risk factor}

The term risk factor has been in use only since the 1960s (2). Given this short history, its general meaning of an exposure that is statistically related in some way to an outcome has caught on pretty well. That smoking is a risk factor for cardiovascular disease and lung cancer is almost universally known and fairly well understood among educated people. But usage of the term risk factor in the literature can still be surprisingly imprecise. In Last's Dictionary of Epidemiology (2), a risk factor is defined as:

...an aspect of personal behavior or lifestyle, an environmental exposure, or an inborn or inherited characteristic which on the basis of epidemiological evidence is known to be associated with healthrelated condition(s) considered important to prevent.

That is a broad and rather vague definition that leaves unanswered the issues of causal role, strength of association, and modifiability. In an effort to clarify the issue, Beck (3) listed a definition that was adopted for the World Workshop on Periodontics in 1996:

Risk factor: an environmental, behavioral, or biologic factor confirmed by temporal sequence, usually in longitudinal studies, which if present directly increases the probability of a disease occurring, and if absent or removed reduces the probability. Risk factors are part of the causal chain, or expose the host to the causal chain. Once disease occurs, removal of a risk factor may not result in a cure.

This is a more complete definition. Its key contributions are: (a) the emphasis on the the temporal sequence of exposure before outcome; (b) acknowledgement that a risk factor is part of the causal chain; and (c) the acceptance that risk factors are involved in disease onset, not necessarily in its future progression or resolution. The definition does not specify whether a risk factor has to be modifiable, but general usage today says that it does. So smoking is a risk factor, age is not (demographic risk factors is one term used for the nonmodifiable risk factors of age, gender, and race/ethnicity).

Any definition of risk factor must clearly establish that the exposure has occurred before the outcome, or before the conditions are established that make the outcome likely. This, in turn, means that prospective studies are necessary to demonstrate risk factors. However, there are many situations in biomedicine, and certainly in dentistry, where this has not been done, and indeed where it is unlikely that longitudinal studies ever will be done. In these circumstances, an exposure which is associated with an outcome only in cross-sectional data is called a risk indicator. A risk indicator may be a probable risk factor, but caution is needed because crosssectional relationships can be deceptive. (A cross-sectional study is defined as one in which exposure and outcome data are collected at the same time, so the temporal association usually cannot be specified.)

So to summarize this issue, an aspect of modern terminology is that the term risk factor implies causality, and the term should be applied only when the time sequence has been established by prospective studies. When we need to impute risk from cross-sectional data, the term risk indicator should be used.

In the context of risk, the concepts of: (a) social determinants of health, and (b) population health merit some consideration. Social determinants of health imply that we need to broaden our views of risk factors for caries beyond biological entities to encompass the broader social environment, and population health implies that we should look at whole populations as entities, rather than just as collections of individuals. 


\section{Social determinants of health}

After years of identifying and quantifying biological risk factors, researchers have become aware that there is more to disease than just biology. A solid body of evidence now exists to show that there are social, psychological, and even spiritual dimensions of health and disease that must be recognized along with biology (4). Social determinants that have been investigated include such factors as the quality of housing, availability of public transport, crime levels, street lighting, access to parks and open space, and the degree of neighborhood social cohesion and emotional support that comes with normal human contact. Poor social circumstances are linked to disease by way of material, psychosocial, and behavioral pathways. Social and environmental disadvantages, even of quite a subtle kind, can lead directly to poor health behavior and subsequent biological disturbances. As examples, the role of loneliness as a risk factor for coronary disease has been known for some time (5), and high income differentials in an area of low income can lead to excess mortality when compared to areas of high income with lower differentials (6). Living in a poverty area in an otherwise well-to-do society increases the risk of bad health outcomes (7). The long-term study of interventions in coronary heart disease in North Karelia, Finland, has increased our knowledge on risk factors for coronary heart disease at all levels. Reduction in risk factors, especially smoking, has led to reduction in mortality, but unexplained regional differences still persist (8). (This latter finding is a population health issue.)

This argument therefore holds that social stresses in themselves can negatively affect health (9). As one example, the gap in cardiovascular disease rates, measured as both mortality and morbidity, between western European countries and those that were formerly part of the Soviet bloc were accentuated sharply around the time of the breakup of the Soviet Union. This phenomenon has been attributed to the high degree of social stress that accompanied the breakup (10).

Dental research in this area has not developed to the extent it has in medical research, but there are indications that similar relations exist between social determinants and oral disease. Parents' employment status and attitudes have been identified as determinants of the dental health of young children in Belfast (11), and work stress was found related to oral health among workers in Brazil (12).
Caries can be considered a social disease, given that it has been related to neighborhood characteristics like the percentage with public rather than private housing, rates of poliomyelitis vaccination, car ownership, and degree of financial problems (13). While dental researchers have for years used socioeconomic status, or social class (SES) as a stratifying variable in their analyses, SES is usually some mix of years of education, current income, or occupation held. British researchers in recent years have led the way in expanding that rather narrow dimension into one which incorporates a broader social view of deprivation (14).

If diseases such as coronary heart disease have a social dimension, why should not the oral diseases also have social determinants? After all, there are common risk factors for coronary disease and oral diseases (15). What this broader view of disease brings home to us is that while biological risk factors are active at one level, there is this broader, macro-level social setting which mediates the disease process in ways not yet well understood. We need more research into this area, and that leads us to population health.

\section{Population health}

Population health is a broader concept than either public health or community health. Its perspective, as stated in a Canadian government document, is to address the entire range of factors that affect health, rather than focusing on specific risks and clinical factors related to particular diseases (16). This is an excellent concept, though it presents challenges for research design. Part of it means to focus on the social structures and social processes within which all ill-health originates, rather than on individual and isolated risks.

The concept of a risk factor is generally understood to apply to individuals. To use cardiovascular disease as an example, the major risk factors for heart disease in individuals are well known: family history, high-fat diet, high blood cholesterol (low-density lipoprotein), hypertension, high blood triglycerides, sedentary lifestyle, and of course smoking. (Blood homocysteine and C-reactive protein levels may join this list soon.) These risk factors were identified over years of research in community-based prospective studies like those in Framingham, Massachusetts (17) and North Karelia, Finland (18). Nowadays, these risk factors are standard items for prevention of heart disease 
in health-promotion activities. Apart from family history and genetic inheritance, these are all modifiable risk factors at individual level, and most research in this area has focused on the relative strength of the risk factors.

How does the concept of population health apply in oral health? Some start has been made by British researchers in assessing the relationship between social deprivation and oral health. SES, or social class, has long been included in such models, but while SES is recognized as a complex variable, our view of it has historically been rather narrow. It usually comes with the assumption that lower education means less understanding of disease-preventive behavior, and the implication is therefore that more education will lead to improved behavior. Recent studies from Britain have shown that caries levels are related to the degree of social deprivation in a geographic area (e.g. an electoral ward) (14, 19-21). Social deprivation is a broader concept than just SES and takes into account some aspects of population health. As an example, the Jarman score, one of several indexes used to measure area deprivation, uses eight social factors available from census data that were reported by medical practitioners to be those that most increased their workload (22). Some of these factors are elderly people living alone, unemployed people, overcrowded households, and households with children under 5 years of age. Interestingly enough, the Jarman measure was developed by the National Health Service for planning purposes and for weighting capitation payments to general medical practitioners. Even so, it works well as a measure of deprivation for small areas, and can be readily matched with other data.

Study designs in population health lean heavily on the ecological design, i.e. one in which the population itself is the unit of study. Ecological designs have long been criticized on the grounds of the 'ecologic fallacy,' i.e. when a population is the unit of study, those with the outcome may not necessarily be the same people who received the exposure. However, we should not forget that Dean's 21-city study, upon which the validity of fluoridation is based, was an ecologic design (23, 24). In fact the impact of a population measure like water fluoridation can only be assessed through ecological approaches, as can the effects of environmental impacts like the Chernobyl radiation disaster in 1986 (bad) or use of unleaded gasoline (good).

\section{Risk factors for caries}

Despite great advances in our understanding of dental caries over the last generation, our knowledge of the role of risk factors in caries is not as precise as we would like it to be. Traditionally, we think of issues like mutans streptococci counts, consumption of sugars, oral hygiene, and exposure to fluorides. These are all 'biological' risk factors, i.e. factors operating within the oral cavity, and we have tended to assess their effects individually. But while all of these factors are involved to some extent in caries development, caries is too complex a disease to let us expect that any one risk factor will serve as an accurate predictor of future disease. With cariogenic bacteria, for example, it has long been assumed that a high count of mutans streptococci in a patient is a risk factor for caries $(25,26)$. But while the causative role of these bacteria in caries is unquestioned, the direct association between bacterial counts and caries incidence is found only at the group or population level, and not at the individual level (27). Even then, the strength of the association is often modest. For the most part, bacteriological tests are highly specific (28), which means that a low mutans streptococci count accurately predicts low caries experience. However, they also have low positive predictive value, meaning that a lot of people with high mutans streptococci counts do not develop caries.

High consumption of sugars or other fermentable carbohydrates has been known to be an etiological factor in caries for decades, yet a systematic review has shown that in the modern age of frequent fluoride exposure, the relationship between sugar consumption and caries experience is not consistent (29). This means that a lot of people are eating a lot of sugar without getting caries. With oral hygiene, there is usually an association between better oral hygiene and lower caries experience at the group or population level, but again positive predictive value is low, i.e. many children with poor oral hygiene do not have much caries.

Current efforts in risk assessment is to develop models in which these risk factors, and others, are put together to get a combined explanatory effect. But the complete answer has not yet been found, and why we do not have a better understanding of the risk factors for caries, and their interactions, is an intriguing question (30). I would suggest that it is because the number of prospective, 
community-based studies that have assessed caries risk is really rather small, and with the small number of studies on different populations (31-33) it is not surprising that the results do not always agree with each other.

\section{Prevention targeted at the high-risk individual}

Caries is no longer universal in schoolchildren, and the distribution in the high-income countries now looks like that shown in Fig. 1. These data are not new; they come from a study of a representative sample of schoolchildren in the US, but they illustrate well the skewed distribution of caries that we see in all the high-income countries today. It is this distribution, where we find that $20 \%$ of the 15 -year olds have about $60 \%$ of all the caries, that has led to our interest in targeted prevention. On the face of it, it makes sense to say that we should concentrate our resources on those individuals (the 'high-risk' group) at the bottom of the distribution who are in most need. However, this concept contradicts the population approach espoused by Rose (34). Rose, using mortality from cardiovascular disease as his outcome, concluded that it is more efficient to move the whole distribution curve to the left rather than focus on the high-risk individuals at the right-hand end of the distribution curve. Population approaches for dental caries would include water fluoridation, restriction of

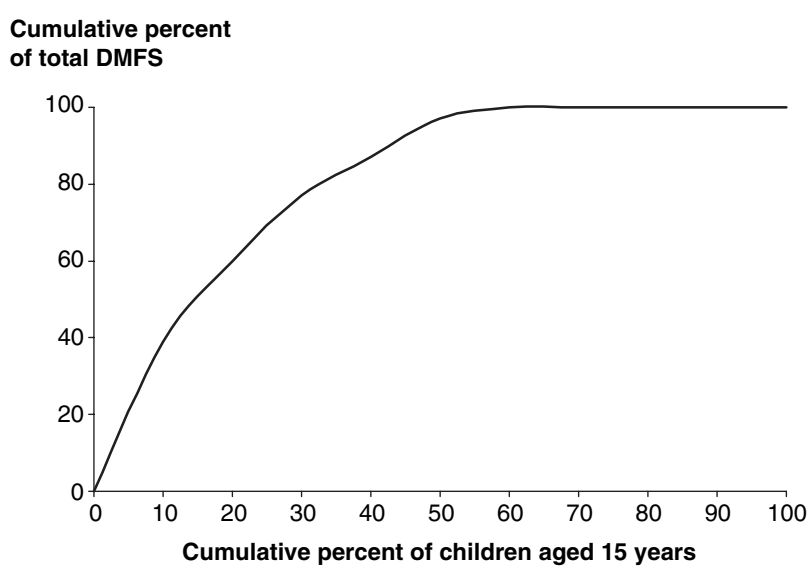

Fig. 1. Cumulative frequency distribution of the proportion of total DMF teeth in schoolchildren aged 15 years, US 1988-94. Source: US Department of Health and Human Services, National Center for Health Statistics. Third National Health and Nutrition Examination Survey, 1988-94. Public Use Data File No. 7-0627. Hyattsville, MD, CDC, 1997. sugar intake by regulation or financial incentives, and dental health education through the mass media.

Despite Rose's arguments, targeting still has intuitive appeal. However, there are several caveats to be considered, the first of which is identifying the high-risk individuals. Targeting has been usually thought of as working at the individual level. For example, some predictive test will be used in screening and the $25 \%$ or so of the children identified as 'high-risk' are then subject to more intensive prevention activities than the other threequarters of the group. However, as discussed above and analyzed by others, there is no clearly superior method for identifying these children (30). In addition, schools dislike programs in which some children will be chosen to participate and some will not. A final problem with targeting to individuals is that it may not work $(35,36)$; too many of these identified children are not compliant and seem to have little interest in their oral health. It seems likely that those not interested in their oral health constitute a hard-to-reach group who also do not respond well to other health-promotion activities.

A further problem with targeting individuals in public health is that the utility of models intended to predict risk in individuals has to be questioned when they require expensive and time-consuming tests to collect data for some of the parameters in the model. Clearly, if a predictive method is to be useful it has to be quick and inexpensive, as well as valid and reliable. It is perhaps rather depressing to realize that after all of the first-class research that has led to sophisticated prediction models, a clinician's judgment of whether a child is likely to develop further caries or not is still one of the most reliable predictors we have (37). When we add this sobering fact to the consistent finding that the best predictor of future caries is past caries, we can suggest again (38) that a good discriminator of caries risk that a private practitioner can use is the presence of approximal caries.

Children with at least one approximal lesion in a permanent tooth, whether treated or not, have higher DMF scores that do children without approximal lesions. This is shown in Fig. 2, with data from a nationwide study of a representative sample of the US population. Data in Fig. 2 are for children aged 12-17 years, and it shows that children with at least one approximal lesion have higher DMFT scores at all ages than children who do not. Children with at least one approximal 


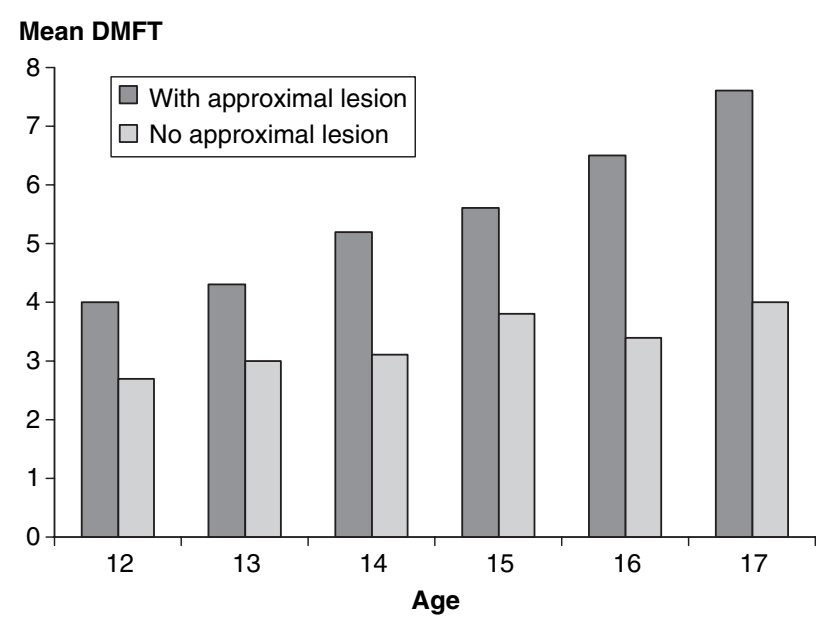

Fig. 2. Mean DMFT scores for US children aged 12-17 years for those with at least one approximal lesion and those with no approximal lesion. Source: US Department of Health and Human Services, National Center for Health Statistics. Third National Health and Nutrition Examination Survey, 1988-94. Public Use Data File No. 7-0627. Hyattsville, MD, CDC, 1997.

lesion, as a proportion of those with any DMFT score above zero, ranged from $13.4 \%$ of 10 -year olds to $35.6 \%$ of 17 -year olds. These data suggest that the presence of at least one approximal lesion in a permanent tooth is a fairly good marker for high-risk status. It identifies around one-quarter of American children as being at high risk, and it requires no counts, samples, or tests.

\section{Prevention targeted at the high-risk community}

As an alternative to targeting programs at individual children in a school, the high-risk community can be the recipient. This approach, which I call geographic targeting, is something between a whole-population approach and individual targeting. As mentioned earlier, we know that social deprivation is associated with caries, and just about any public health jurisdiction has some areas of social deprivation within its borders. The data needed to identify socially deprived areas is usually readily available to the public, for example in census data. In the US, the data for the proportion of children in each school who are eligible for a free or subsidized lunch, for all school districts in the country, is available on a public website. A dental administrator can then simply look up the school districts in his or her jurisdiction. The higher the proportion of children eligible for free or subsidized lunch, the greater the degree of poverty. A major attraction of this approach to the dental administrator is that there are virtually no administrative costs to doing this; someone else has already collected and filed this information.

In the US, resources for dental public health programs are more restricted than they are in Europe, and hence efficiency in using these resources is essential. In recent years, there has been substantial growth in school-based sealant programs conducted by state and local health departments, and the experiences from two of these programs, one in Ohio and one in New York, are instructive in looking at targeting. The dental directors in both states coordinate sealant teams that use portable equipment and move from school to school to treat the children. Each team comprises hygienists and assistants, and the law usually requires that a dentist screen the children first to prescribe sealants. No program has sufficient resources to go to all schools in a state; Ohio is one of a number of states that targets its sealant program at specific schools, which are chosen for the proportion of children eligible for free or subsidized lunch. Ohio does not attempt to select out individual 'high-risk' children from these schools; all children in selected schools are eligible for treatment where indicated. This is an example of true geographic targeting - the whole population is eligible within specific geographic boundaries. Evaluation results show that the Ohio program substantially reduces the disparity in sealant placement between children from lowincome families and those from higher-income families (39).

New York also selects geographic areas for its sealant program, the choice being based upon SES, demonstrated need, community interest, operational feasibility and ability to comply with the health department's rules and regulations (40). It differs from the Ohio program in that individual children are selected by the screening dentist as high risk on the basis of previous dental caries and deep pits and fissures, so this program combines some aspects of geographic targeting and individual selection. What an evaluation showed, however, is that the criteria for selection of children and teeth were not well adhered to by the sealant team (40). This finding did not indicate a cavalier disregard for the carefully designed criteria on the part of the sealant team, but rather a necessary response to pressures from parents and school personnel that the sealant teams found difficult to resist. It indicates yet again that targeting of 
individuals is just difficult to do in everyday situations.

\section{Conclusions}

The concept of risk factors for disease is spreading from the purely biological factors, with perhaps SES added in, to become more sensitive to inclusion of social factors. Some progress has been made, and further research will identify which of these indicators are the most important, and most practical for an administrator to use, in predicting geographic areas of high dental caries risk.

Population strategies are the basis for all dental public health programs, for example water fluoridation and dental health education. These are programs aimed at a whole population, regardless of individual risk, and whose effectiveness is best measured at population level. Identifying and then targeting individuals at high risk is not a recommended strategy in public health because the risk assessment models are far from precise at individual levels, and there are practical problems in treating the identified individuals successfully. Between these two approaches is geographic targeting, in which schools, or school districts, or even whole counties can be identified as being at high risk. If this can be done with easily-available data then there are no administrative costs to identifying the targeted areas. With the skewed distribution of caries that is seen in all the high-income countries today, this combination of some population-based preventive measures and some geographic targeting seems the best way to proceed to reduce the burden of caries in our children even further.

\section{Acknowledgement}

I am indebted to Dr Robert Lubwama for his assistance with data analysis.

\section{References}

1. Rothman KJ. Epidemiology: an introduction. New York: Oxford University Press; 2002.

2. Last JM, ed. A dictionary of epidemiology. 4th edn. New York: Oxford University Press; 2001.

3. Beck JD. Risk revisited. Community Dent Oral Epidemiol 1998;26:220-5.

4. Marmot M, Wilkinson RG, editors. Social determinants of health. New York: Oxford University Press; 1999.
5. Kaplan GA. Social contacts and ischaemic heart disease. Ann Clin Res 1988;20:131-6.

6. Lynch JW, Kaplan GA, Pamuk ER, Cohen RD, Heck $\mathrm{KE}$, Balfour JL, et al. Income inequality and mortality in metropolitan areas of the United States. Am J Public Health 1998;88:1074-80.

7. Yen IH, Kaplan GA. Poverty area residence and changes in depression and perceived health status: evidence from the Alameda County Study. Int J Epidemiol 1999;28:90-4.

8. Jousilahti P, Vartiainen E, Tuomilehto J, Pekkanen J, Puska P. Role of known risk factors in explaining the difference in the risk of coronary heart disease between eastern and southwestern Finland. Ann Med 1998;30:481-7.

9. Brunner E, Marmot MG. Social organization, stress, and health. In: Marmot M, Wilkinson RG, editors. Social determinants of health. New York: Oxford University Press; 1999. p. 17-43.

10. Bobak M, Marmot M. East-West mortality divide and its potential explanations: proposed research agenda. BMJ 1996;312:421-5.

11. Freeman R, Breistein B, McQueen A, Stewart M. The dental health status of five-year-old children in north and west Belfast. Community Dental Health 1997;14:253-7.

12. Marcenes WS, Sheiham A. The relationship between work stress and oral health status. Soc Sci Med 1992;35:1511-20.

13. Gratrix D, Holloway PJ. Factors of deprivation associated with dental caries in young children. Community Dental Health 1994;11:66-70.

14. Jones CM, Woods K, Taylor GO. Social deprivation and tooth decay in Scottish schoolchildren. Health Bull 1997;55:11-5.

15. Sheiham A, Watt RG. The common risk factor approach: a rational basis for promoting oral health. Community Dent Oral Epidemiol 2000;28:399-406.

16. Young TK. Population health; concepts and methods. New York: Oxford University Press; 1998.

17. Castelli WP. Epidemiology of coronary heart disease: the Framingham study. Am J Med 1984;76:4-12.

18. Salonen JT. Prevention of coronary heart disease in Finland-application of the population strategy. Ann Medicine 1991;23:607-12.

19. Jones C, Taylor G, Woods K, Whittle G, Evans D, Young P. Jarman underprivileged area scores, tooth decay and the effect of water fluoridation. Community Dental Health 1997;14:156-60.

20. Jones CM, Worthington $H$. The relationship between water fluoridation and socioeconomic deprivation on tooth decay in 5-year-old children. $\mathrm{Br}$ Dent J 1999;186:397-400.

21. Jones CM, Taylor GO, Whittle JG, Evans D, Trotter DP. Water fluoridation, tooth decay in 5 year olds, and social deprivation measured by the Jarman score: analysis of data from British dental surveys. BMJ 1997;315:514-7.

22. Jarman B. Underprivileged areas: validation and distribution of scores. BMJ 1984;289:1587-92.

23. Dean HT, Jay P, Arnold FA Jr, Elvove E. Domestic water and dental caries. II. A study of 2,832 white children aged 12-14 years, of eight suburban Chicago communities, including $L$. acidophilus studies of 1,761 children. Public Health Rep 1941;56:761-92. 
24. Dean HT, Arnold Jr FA, Elvove E. Domestic water and dental caries. V. Additional studies of the relation of fluoride domestic waters to dental caries experience in 4,425 white children aged 12-14 years of 13 cities in 4 states. Public Health Rep 1942;57:1155-79.

25. Krasse B. Biological factors as indicators of future caries. Int Dent J 1988;38:219-25.

26. Stecksen-Blicks C. Lactobacilli and Streptococcus mutans in saliva, diet and caries increment in 8- and 13-year-old children. Scand J Dent Res 1987;95:18-26.

27. Kingman A, Little W, Gomez I, Heifetz SB, Driscoll WS, Sheats R, et al. Salivary levels of Streptococcus mutans and lactobacilli and dental caries experiences in a US adolescent population. Community Dent Oral Epidemiol 1988;16:98-103.

28. van Houte J. Microbiological predictors of caries risk. Adv Dent Res 1993;7:87-96.

29. Burt BA, Pai S. Sugar consumption and caries risk: a systematic review. J Dent Educ 2001;65:1017-23.

30. Hausen H. Caries prediction - state of the art. Community Dent Oral Epidemiol 1997;25:87-96.

31. Beck JD, Weintraub JA, Disney JA, Graves RC, Stamm JW, Kaste LM, et al. University of North Carolina Caries Risk Assessment Study: comparisons of high risk prediction, any risk prediction, and any risk etiologic models. Community Dent Oral Epidemiol 1992;20:313-21.

32. Leverett DH, Proskin HM, Featherstone JD, Adair SM, Eisenberg AD, Mundorff-Shrestha SA, et al.
Caries risk assessment in a longitudinal discrimination study. J Dent Res 1993;72:538-43.

33. Grindefjord M, Dahllof G, Nilsson B, Modeer T. Stepwise prediction of dental caries in children up to 3.5 years of age. Caries Res 1996;30:256-66.

34. Rose G. The strategy of preventive medicine. New York: Oxford University Press; 1992.

35. Seppä L, Hausen H, Pollanen L, Karkkainen S, Helasharju K. Effect of intensified caries prevention on approximal caries in adolescents with high caries risk. Caries Res 1991;25:392-5.

36. Hausen H, Karkkainen S, Seppä L. Application of the high-risk strategy to control dental caries. Community Dent Oral Epidemiol 2000;28:26-34.

37. Graves RC, Abernathy JR, Disney JA, Stamm JW, Bohannan HM. The University of North Carolina Caries Risk Assessment Study. III. Multiple factors in caries prevalence. J Public Health Dent 1991;51:13443.

38. Burt BA. Prevention policies in the light of the changed distribution of dental caries. Acta Odontol Scand 1998;56:179-86.

39. Siegal MD, Miller DL, Moffat D, Kim S, Goodman P. Impact of targeted, school-based dental sealant programs in reducing racial and economic disparities in sealant prevalence among schoolchildren - Ohio, 1998-1999. MMWR 2001;50:736-8.

40. Kumar JV, Wadhawan S. Targeting dental sealants in school-based programs: evaluation of an approach. Community Dent Oral Epidemiol 2002;30:210-5. 